American Journal of Optics and Photonics
2016; 4(5): $51-56$
http://www.sciencepublishinggroup.com/j/ajop
doi: 10.11648 /j.ajop.20160405.13
ISSN: $2330-8486$ (Print); ISSN: $2330-8494$ (Online)

\title{
Electrons Break to Photons Even in a Low Voltage Electric Circuit
}

\author{
Hadi Ensan \\ Technical College, Technical and Vocational University, Neyshaboor, Iran
}

\section{Email address:}

h_ensan1@yahoo.ca

\section{To cite this article:}

Hadi Ensan. Electrons Break to Photons Even in a Low Voltage Electric Circuit. American Journal of Optics and Photonics.

Vol. 4, No. 5, 2016, pp. 51-56. doi: 10.11648/j.ajop.20160405.13

Received: October 26, 2016; Accepted: November 11, 2016; Published: December 12, 2016

\begin{abstract}
This paper is the theory of breaking electrons in ordinary circuit elements like resistors and Light-Emitting Diodes (LED's). Undergoing a change of electron has not been considered in the low voltage circuits so far. As it is shown here, there is a difference current before and after LED and resistors. The possibility of leakage current or escaping of electrons from the circuit to make electrostatic charges also considered and tested for LED. It is concluded that the reverse action of Photoelectric effect $\left(e V_{o}=h f-\varphi\right)$, creating energy from mass of electrons are happened not just in a sophisticated high energy accelerators but in daily life electric circuits. Referring to this paper, a large number of missing electrons break to photons, although the drift velocity of electrons is very low. Under going a change of electrons has been considered in the circuits of these experiments. According to the Kirchhoff second rule called the junction rule, the conservation of charge implies the junction rule, because charge does not originate or accumulate or annihilate at this point. It expresses that in any closed single loop electric circuit there is no source or well of charge besides the power supply and the current remains constant at all points of the circuit. Based on the results of this paper and missing some part of current, the conservation of charge does not show high accuracy in this paper.
\end{abstract}

Keywords: Electrons Produce Photons, Charge Rule Violation, Elementary Particle

\section{Introduction}

The interaction between electrons and photons has already been investigated [1] and $e^{-}+e^{+} \rightarrow \gamma+\gamma$, Annihilation of electron- positron pair that produces a pair of gamma rays has been studied [2]. We know that photons or $\gamma$-rays can undergo three major types of collision. Striking an electron, losing all its energy to it and simply disappearing (Photoelectric effect) [3] and [12], or a photon can collide with an electron and be scattered to one side (Compton Effect). The third way in which a photon can be stopped is through a process called pair production that in this process, mass is created from the photon's energy [9]. The track of electron has already been viewed in the alternating- gradient synchrotron at Brookhaven National Laboratory in US at the Bubble-chamber by photograph of electron- positron pair formation [10]. In this paper the reverse action of photoelectric effect is studied. In pair production electronpositron are produced by $\gamma$-rays or by interacting the incident beam that is composed of antiprotons with a hydrogen nucleus (proton). In reverse process the collision of electronpositron results production of $\gamma$-rays. In all of cases, the Conservation of charge is valid. In the experiments mentioned here, it seems that rule to be violated. Although a battery pack of a pocket calculator with the voltage of $3.0 \mathrm{~V}$ delivers a current of $0.17 \mathrm{~mA}$ [4] which seems to be low, but for these experiments lower amperage is suggested. For more accurate results it is recommended that:

1) The better digital Multi meters especially more precise Ammeters with the Ranges of $(\mu A, n A)$ with Higher Resolutions and Better Accuracy, DC power supply with constant voltage, etc., are needed. The Ammeter is suggested to be with selector of high amperage with the range of Micrometer or Nano ammeter, for example if we could read the current of $5.76 \mathrm{~A}$ in the range of $\mu \mathrm{A}$, it could be as $5,763,235.07 \mu \mathrm{A}$ or at the range of $\mathrm{nA}$ may be 5,763,235,072.3 $n A$. Although the three first digits are the same but by increasing the resolution, the differences of measurements appear better.

2) More powerful resistor or light equipment in the circuit to be used to find more amperage differences. 
3) The grounding wiring system should have the lowest possible resistant to the ground for measuring the possibility of lowest leakage current of the circuit to the surrounding environment.

\section{Procedure}

\subsection{Material List}

Preparing a circuit with safety measures with the items and material list (Figure 1) including:

a) A direct current supply having ranges of 3, 6, 9, and 12 Volts or using a proper DC voltage convertor.

b) At least four digital Multimeters including: one Micro ammeter and the other possessing the selectors of Milli ammeter and Millivolt meter.

c) A LED bar light about power nominal of 2 Watts.

d) A metallic tray as a heat sink and a protective shield.

\subsection{Preparing the First Test Circuit for Measurements}

In this experiment at first two digital ammeters in parallel connected to one side and one digital ammeter connected to another side. For the next step the position of ammeters are interchanged. A voltmeter is also used for measuring the circuit voltage.

Connecting the circuit parts according to Figure 1 and reversing the direction of LED bar light to inside for preventing of damage to eyes from its glare. It is suggested even cover it with a noun combustible semi-transparent sheet for reducing its reflected glare from tray and do not touch the light bar when is on with bare hand for not burning the hand. At the positive side of LED two ammeters are connected parallel to each other and at negative side of light bar an ammeter is connected in series in the circuit. The range of one of ammeter at positive side is $\mathrm{A}$ and the other is $\mu \mathrm{A}$. The range of the negative side is A. After each measurement and the circuit was disconnected for time lapse of 20 seconds and then turned on, 10 seconds waited for stabilizing the circuit parameters, the amperage was measured. The tests repeated for 5 times, then the two ammeters connected to the positive side of light bar moved to the negative side and the ammeter connected the negative side moved to the positive side. The measurements repeated as table1.

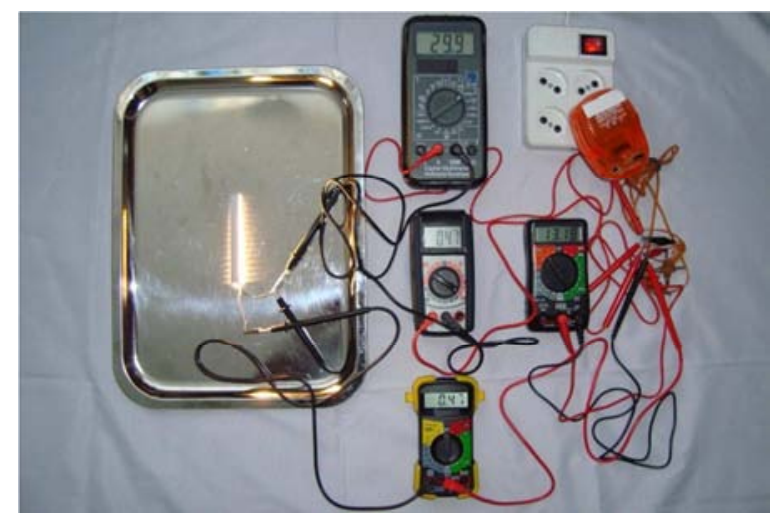

Figure 1. The circuit prepared and taken picture by author Ensan for current measurement before and after LED. The upper and middle left ammeters (Ampere meters) connected parallel to measure the current of one side of light bar and the lower ammeter measures current of another side of LED. There is difference between them. At the middle right a voltmeter measures the circuit voltage. Light bar placed inverse on the tray for avoiding its glare to eyes.

\subsection{Tests and the Tables of the Data}

Preparing the tables with the columns of $I_{1}(A)$ for one side, $I_{3}(\mu A)$ for the same side and $I_{2}(A)$ for another side of LED bar, $\Delta I(\mu A), P(w), \Delta P\left(10^{-6} w\right)$ And carrying out the measurements and noting the results:

Table 1. The five first rows are related to the current measurement of positive side of LED bar and the five next rows are related to the current measurement at its negative side. The current difference between them ( The difference of second five average current of negative side (29.98 $\mu A$ ) and the first five average of current of positive side; $(28.96 \mu \mathrm{A})$ is $\Delta I=1.02 \mu \mathrm{A}$. This shows some electrons break while passing through the LED light.

\begin{tabular}{|c|c|c|c|c|c|c|c|}
\hline Test Number & $I_{1}(A)$ & $I_{2}(A)$ & $I_{3}(A)$ & $\Delta I(\mu A)$ & $V(V)$ & $P(W)$ & $\Delta P(\mu W)$ \\
\hline 1 & 0.46 & 0.45 & 28.8 & 28.8 & 13.06 & 5.942 & 376.12 \\
\hline 2 & 0.46 & 0.46 & 29.4 & 29.3 & 13.06 & 6.007 & 383.96 \\
\hline 3 & 0.46 & 0.46 & 29.3 & 29.3 & 13.06 & 6.007 & 382.658 \\
\hline 4 & 0.46 & 0.46 & 29.1 & 29.1 & 13.05 & 6.003 & 379.755 \\
\hline 5 & 0.45 & 0.45 & 28.2 & 28.2 & 12.99 & 5.845 & 366.318 \\
\hline Average & 0.458 & 0.457 & 28.96 & 28.96 & 13.044 & 5.978 & 377.762 \\
\hline 6 & 0.47 & 0.47 & 30.4 & 30.4 & 13.25 & 6.227 & 402.8 \\
\hline 7 & 0.475 & 0.47 & 30.6 & 30.6 & 13.24 & 6.289 & 405.144 \\
\hline 8 & 0.47 & 0.47 & 30.1 & 30.1 & 13.16 & 6.185 & 396.116 \\
\hline 9 & 0.47 & 0.47 & 30.1 & 30.1 & 13.14 & 6.175 & 395.514 \\
\hline 10 & 0.475 & 0.47 & 30.5 & 30.5 & 13.21 & 6.208 & 402.905 \\
\hline Average & 0.472 & 0.47 & 30.34 & 30.34 & 13.20 & 6.218 & 400.495 \\
\hline \multirow{2}{*}{\multicolumn{7}{|c|}{$\begin{array}{l}\text { *Proportional Amperage and Power } \\
\text { *The Average Difference of Losing Power Because of Breaking Electrons }\end{array}$}} & 391.077 \\
\hline & & & & & & & 13.315 \\
\hline
\end{tabular}

For a regular circuit and non sensitive ammeters, connecting one ammeter before and another after the resistor, they do not show any difference of the current in a single loop circuit. Although in an ionic solution, both positive and negative charges contribute to the current by moving in opposite directions [5] but current in a copper wire is just related to the moving of electrons.

\subsection{Analyzing the Results of Table 1}

As a check on these results we find that the difference 
between the last column two averages

$$
\Delta P=13.315 \mu W
$$

For better comparison between the result numbers, the first average voltage is supposed to be equal to the last average voltage and its proportional amperage, power and the average difference of losing power are calculated.

There is possibility that the amperage difference has leaked to the environment. There is possibility that Also charge do accumulate on the surface of conductors. The surface area of the conductors that are used in common circuits are much too small to accumulate a significant amount of charge [6]. The leakage current test (2.7.) is done for it and does not show a measurable leakage current. In 2.9. an example of mathematical analyzing is introduced.

The result shows negative side current is more than the positive side in the single loop circuit. Although based on classical Physics principles could not be a well that electrons fall down it, but is considered that some part of electrons missing. The interpretation could be that at least part of missing electrons break to photons.

\subsection{Considering the Relation Between Voltage and the Missing Current}

Using the same circuit of Figure 1 and repeating the different voltages give us the difference current for different voltage. The results of measurements are shown in table 2 . For all tests indicated in negative side of LED the Multi meters measure $I_{1}(A)$ and $I_{3}(\mu A)$ at the negative side and the Multi meter measures $I_{2}(A)$ located on positive side. At left side of the table 2(positive side) two Multi meters measuring $I_{1}(A)$ and $I_{3}(\mu A)$ placed on positive side and Multi meter measures $I_{2}(A)$ located on negative side.

Table 2. Illustrates the relation between applied voltage of LED and the difference current on the negative and positive side of it. By increasing the voltage the missing current also increases. The difference currents indicated with* on the positive side are proportional amounts if their voltages were as the same as negative side.

\begin{tabular}{|c|c|c|c|c|c|c|c|c|c|c|}
\hline \multirow[b]{2}{*}{ \# } & \multicolumn{5}{|c|}{ Negative Side } & \multicolumn{5}{|c|}{ Positive Side } \\
\hline & $I_{1}(A)$ & $I_{2}(A)$ & $I_{3}(A)$ & $V(V)$ & $\Delta I_{1}(\mu A)$ & $I_{1}(A)$ & $I_{2}(A)$ & $I_{3}(A)$ & $V(V)$ & $\Delta I_{1}(\mu A)$ \\
\hline 1 & 0.06 & 0.06 & 8.2 & 8.62 & 8.2 & 0.05 & 0.05 & 6.7 & 8.62 & 6.68 \\
\hline 2 & 0.05 & 0.05 & 7.5 & 8.61 & 7.5 & 0.05 & 0.05 & 6.6 & 8.58 & 6.6 \\
\hline Average & & & & 8.615 & 7.85 & & & 6.65 & $* 8.615$ & $* 6.68$ \\
\hline \multicolumn{11}{|c|}{$\Delta I_{a}=\Delta I_{l}-\Delta I_{2}=7.85-6.64=1.21 \mu \mathrm{A}$ and $V_{a}=8.615 \mathrm{~V}$} \\
\hline 1 & 0.14 & 0.14 & 20.8 & 20.8 & 9.82 & 0.14 & 0.14 & 19.4 & 9.75 & 19.4 \\
\hline 2 & 0.14 & 0.14 & 21.3 & 21.3 & 9.82 & 0.14 & 0.14 & 19.4 & 9.76 & 19.4 \\
\hline Average & & & 21.05 & & 9.82 & & & & $* 9.82$ & $* 19.4$ \\
\hline \multicolumn{11}{|c|}{$\Delta I_{b}=\Delta I_{1}-\Delta I_{2}=21.05-19.4=1.65 \mu \mathrm{A}$ and $V_{b}=9.82 \mathrm{~V}$} \\
\hline 1 & 0.24 & 0.24 & 35.3 & 35.3 & 10.92 & 0.23 & 0.23 & 31.8 & 10.81 & 31.8 \\
\hline 2 & 0.24 & 0.24 & 36.3 & 36.3 & 10.94 & 0.23 & 0.23 & 31.8 & 10.73 & 31.8 \\
\hline Average & & & & 35.8 & 10.93 & & & 31.8 & *10.93 & $* 32.272$ \\
\hline \multicolumn{11}{|c|}{$\Delta I_{c}=\Delta I_{l}-\Delta I_{2}=35.8-32.272=2.728 \mu \mathrm{A}$ and $V_{c}=10.93 \mathrm{~V}$} \\
\hline 1 & 0.45 & 0.45 & 65.5 & 13.07 & 65.5 & 0.43 & 0.43 & 61.8 & 12.87 & 61.8 \\
\hline 2 & 0.45 & 0.44 & 65.5 & 13.10 & 65.5 & 0.43 & 0.43 & 61.4 & 12.90 & 61.4 \\
\hline Average & & & 65.5 & 13.085 & & & & & *13.085 & $* 62.556$ \\
\hline \multicolumn{11}{|c|}{$\Delta I_{d}=\Delta I_{1}-\Delta I_{2}=65.5-62.556=2.944 \mu \mathrm{A}$ and $V_{d}=13.085 \mathrm{~V}$} \\
\hline
\end{tabular}

The results of table 2 show increasing the difference current (missing current) by increasing the circuit voltage.

Let us see if there is a relation between missing current and the applied voltage.

$$
\begin{gathered}
K_{a}=\frac{V_{a}}{\Delta I_{a}}=\frac{8.615 \mathrm{~V}}{1.21 \times 10^{-6} \mathrm{~A}}=7,946,280.99 \Omega \approx 8 M \Omega \\
K_{b}=\frac{V_{b}}{\Delta I_{b}}=\frac{9.82 \mathrm{~V}}{1.65 \times 10^{-6} \mathrm{~A}}=5,951,515.15 \Omega \approx 6 M \Omega \\
K_{c}=\frac{V_{c}}{\Delta I_{c}}=\frac{10.93 \mathrm{~V}}{2.728 \times 10^{-6} \mathrm{~A}}=4,006,598.24 \Omega \approx 4 M \Omega \\
K_{d}=\frac{V_{d}}{\Delta I_{d}}=\frac{13.085 \mathrm{~V}}{2.944 \times 10^{-6} \mathrm{~A}}=4,444,633.15 \Omega \approx 4.4 M \Omega
\end{gathered}
$$

If the K's were equal, we could conclude that the Ohm's Law is ruled for the missing current, but they are not equal.

\subsection{Test for Possibility of Leakage Current}

Material List: (Figure2):

e) Some proper wires, clips and wire connectors f) Proper metallic pot as a leakage current collector testing device.

g) A disconnect switch

h) Electrical insulator sheets like polyethylene and Styrofoam.

i) Ground wire connection for measuring the possibility of accumulated electrons on metallic pot.

\subsection{The Leakage Current Test}

For considering the possibility of leakage current the circuit shown in figure 2, has been prepared. The LED bar placed inside a metallic pot which electrically insulated from the ground. The Multi meter is set to the range of $200 \mu \mathrm{A}$ which is highest resolution in this Multi meter.

Its ampere side is connected momentary to the pot and the common wire connected to the ground wire connection. With the highest voltage tolerable by the bar light $(13.22 \mathrm{~V}$ in this case), the voltage difference of pot and ground measured five times that sometimes for a fraction of second was about between 0.2 to $0.3 \mathrm{mV}$ and Multi meter all the times showed 
current of zero. The waiting time period for possibility of collecting electrons on the surface of pot was 10 minutes before each measurement. The result of possibility the leakage current or escaping electrons from the light bar in these tests is negligible. The conclusion could be that some the missing part of electrons which is the difference between positive and negative sides of light bar have been broken to photons with different frequencies of visible light and infrared, etc.

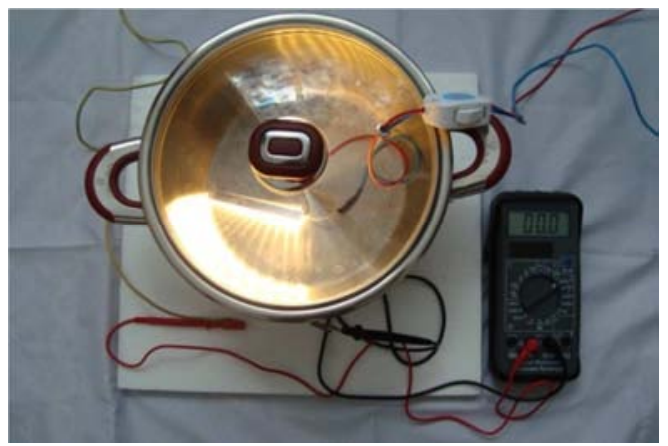

Figure 2. The circuit is prepared and taken picture by author Ensan for possibility of current leakage measurement. The bar light placed inside the metallic pot that is insulated from the ground by 2 layers of polyethylene sheets (not showed in the figure) and a Styrofoam layer of $5 / 8$ inch thickness. The clip of common wire connected to the edge of pot and the wire of current (A) socket connected to the ground wire. Multi meter selector was set to its highest resolution $(200 \mu \mathrm{A})$. The result of current after several times of measurement was zero and the result of relative voltage to ground (has not been shown in this figure) was almost zero (one time showed $0.3 \mathrm{mV}$ ).

\subsection{Measuring of Current Before and After Tiny Electronic Resistors Sides with 1.5V, AA Battery as the Power Supply}

Using two kinds of Multi meters with different ranges and also convertor which its voltage fluctuates make the first test results difficult. In this test a single Multi meter with the highest possible range resolution $(200 \mu \mathrm{A})$ and a single battery AA with nominal voltage of 1.5 Volts is used to cancel the fluctuation of line voltage.

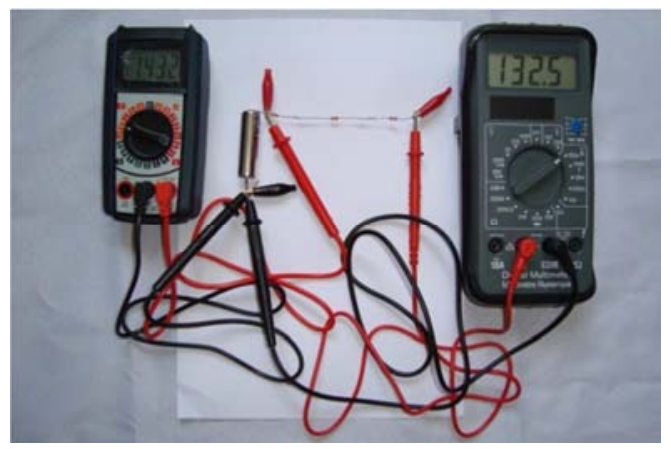

Figure 3. The circuit prepared and taken picture by author Ensan for considering the difference of amperage between the locations of before and after three tiny resistances connected in series with total resistant of 9.81 $K \Omega$. The ammeter shows the current of $132.5 \mu \mathrm{A}$. The average obtained difference current is $\triangle I=40 \mathrm{nA}$. The same experiment is done with the same circuit but instead of $1.5 \mathrm{~V}$, AA battery that figure shows $1432 \mathrm{mV}$, a 9 $V, 6 F 22$ has been used. The average difference amperage obtained $\Delta I=1941$ $n A$.

\subsection{Material and Testing Device List}

a) Three electronic resistances connected together in series with total resistance measurement of:

$$
R_{T}=9.81 \mathrm{~K} \Omega
$$

b) One battery with nominal voltage of $1.5 \mathrm{~V}$, an AA battery

c) One digital Multi meter has set to DC Amperage at the range of $200 \mu \mathrm{A}$

d) One digital Multi meter has set to DC voltage at the range of $2000 \mathrm{mV}$

e) Wires for connections and clips

Connecting the circuit parts according to Figure 3 and measuring the current before and after resistances for five times, yields us the numbers in table 3 :

Table 3. The results of testes for measurements of current before and after of resistances in a single loop circuit. In most cases the current in negative parts (direction of electrons to the resistances) are measured more than that the current of positive side. The numbers singed with * are the average amount of blinking between $132.5 \mu \mathrm{A}$ and $132.6 \mu \mathrm{A}$.

\begin{tabular}{llll}
\hline $\begin{array}{l}\text { Test } \\
\text { number }\end{array}$ & $\begin{array}{l}\text { Voltage } \\
(\boldsymbol{m} \boldsymbol{V})\end{array}$ & $\begin{array}{l}\text { Amperage }(\boldsymbol{\mu} \boldsymbol{A}) \\
\text { At positive side }\end{array}$ & $\begin{array}{l}\text { Amperage }(\boldsymbol{\mu} \boldsymbol{A}) \text { At } \\
\text { negative side }\end{array}$ \\
\hline 1 & 1432 & 132.5 & 132.6 \\
2 & 1432 & 132.5 & 132.5 \\
3 & 1433 & 132.5 & 132.5 \\
4 & 1433 & 132.5 & $132.55^{*}$ \\
5 & 1433 & 132.5 & $132.55^{*}$ \\
Average & 1432.6 & 132.5 & 132.54 \\
\multicolumn{2}{l}{\begin{tabular}{l} 
The Current Difference Between the \\
\multicolumn{2}{l}{ Locations of Before and After Resistances }
\end{tabular}} \\
\hline
\end{tabular}

\subsection{Analyzing the Results of Table 3}

As we already know, in gases and electrolytes which placed in electric field, all negative and positive ions could move to the opposite charge sides. In solid metals in the room temperature like cooper wires in these tests, the only parts of atom could move in the electric field, are the free electrons. The drift velocity of electrons:

$$
V_{d}=\frac{I}{A n_{e}}
$$

In a typical copper wire of radius of $0.815 \mathrm{~mm}$ carrying current of $1 \mathrm{~A}$ is:

$V_{d} \simeq 3.54 \times 10^{-5} \mathrm{~m} / \mathrm{s}$ [7]. Considering the numbers of table 3 and the direction of real current in copper metal in this test that is from negative pole to the positive pole, we obtain:

$$
\Delta I=40 n A=40 \times 10^{-9} A
$$

The current equation was given as:

$\Delta q=\Delta I . t$, for $\mathrm{t}=1$ second, we get:

$$
\Delta q=40 \times 10^{-9} \mathrm{C}
$$

Based on the Einstein equation of mass and energy:

$$
E=m c^{2}
$$

If all of current difference (6) is changed to energy, then the number of electrons that break down to energy is 
obtained from:

$$
N_{e}=\frac{\Delta q}{e}
$$

Where Electron charge

$$
e=1.60219 \times 10^{-19} \mathrm{C}
$$

Substituting in (9) yields the numbers of electrons that annihilate when pass through the resistances:

$$
N_{e}=40 \times 10^{-9} / 1.60219 \times 10^{-19}=2.49658 \times 10^{11}
$$

Electron rest mass:

$$
\begin{gathered}
m_{e}=9.1095 \times 10^{-31} \mathrm{Kg} \\
E=N_{e} \cdot m_{e} \cdot c^{2} \\
E=2.49658 \times 10^{11} \times 1.60219 \times 10^{-31} \times\left(3 \times 10^{8}\right)^{2} \mathrm{~J} \\
E=3.59999 \times 10^{-3} \mathrm{~J}
\end{gathered}
$$

The energy produced by difference current:

$$
\begin{gathered}
W=R \cdot(\Delta I)^{2} . t, \text { for } \mathrm{t}=1 \mathrm{~s} \\
W=9.81 \times 10^{3} \times 40 \times 10^{-9}=3.924 \times 10^{-4} J
\end{gathered}
$$

Comparing (14) and (16) shows that all of missing electrons that come from the current difference have not been changed, but a fraction of them have been changed to photons. We find that fraction from dividing (16) to (14):

$$
K_{r}=\frac{W}{E}=\frac{3.924 \times 10^{-4}}{3.59999 \times 10^{-3}}=10.900=\% 10.9
$$

The total energy dissipating in LED:

$$
W_{t}=R \cdot I_{t}^{2} \cdot t
$$

where, $I_{t}=132.5 \mu A$ and $\mathrm{t}=1 \mathrm{~s}$, then we obtain:

$$
W_{t}=9.81 \times 10^{3} \times 132.5 \times 10^{-9}=1.29982 \times 10^{-3} \mathrm{w}
$$

Dividing (19) to (14) gives the rate of change of electrons to photons in this LED:

$$
K_{t}=\frac{1.29982 \times 10^{-3}}{3.59999 \times 10^{-3}}=0.36106=\% 36.1
$$

This shows for the mentioned condition of the circuit, about 36 percent of total dissipating power comes from the breaking of electrons that are the difference passing current between two negative and positive sides of resistances with the amount of $\Delta q=40 \times 10^{-9} \mathrm{C}$. The rest of dissipating power could be related to kinetic energy of accelerated electrons passing through the resistances when striking to the target atoms and release photons, etc.

\subsection{Measuring of Current Before and After Tiny Electronic Resistors Sides with 9V, 6F22 Battery as the Power Supply}

Repeating the same experiment is done with the same circuit of figure 2, but instead of $1.5 \mathrm{~V}$, AA, another battery with nominal voltage of $9 \mathrm{~V}, 6 \mathrm{~F} 22$ battery has been used. The average difference amperage obtained $\Delta \mathrm{I}=1941 \mathrm{nA}$. The average total circuit voltage is $9.656 \mathrm{~V}$. The same above procedure can be followed for getting the percentage of electrons which disappear and break down to photons.

\subsection{Discussion}

Based on the tests, existence of current difference between two sides of an electric element has not been considered so far. The better results come with higher resolution Multi meters and non-fluctuating voltage supply (constant voltage) and also as table 2 shows the rate of difference current increases by increasing the applied voltage to the element. According to the Kirchhoff's Law, The sum of all the currents coming into a junction must be equal the sum of all the currents leaving it and the Conservation of charge implies the junction rule [6], [8] and [12]. There is difference between the current of coming in and getting out of the LED or resistances for all these experiments. According to our existing knowledge light (photons) come from the accelerated free electrons (like electron beam in a vacuum tube with high voltage electrodes which produces X-rays) or changing the orbits of electrons moving around the nuclei in atoms. Annihilation of matter in collision of electronpositron pair in high energy accelerators produces gamma rays, etc. In these tests some missing electrons produce visible light or infra-red. If electrons could break to photons, therefore, Photon could be one of the sub-building blocks of electron, (Figure 4).

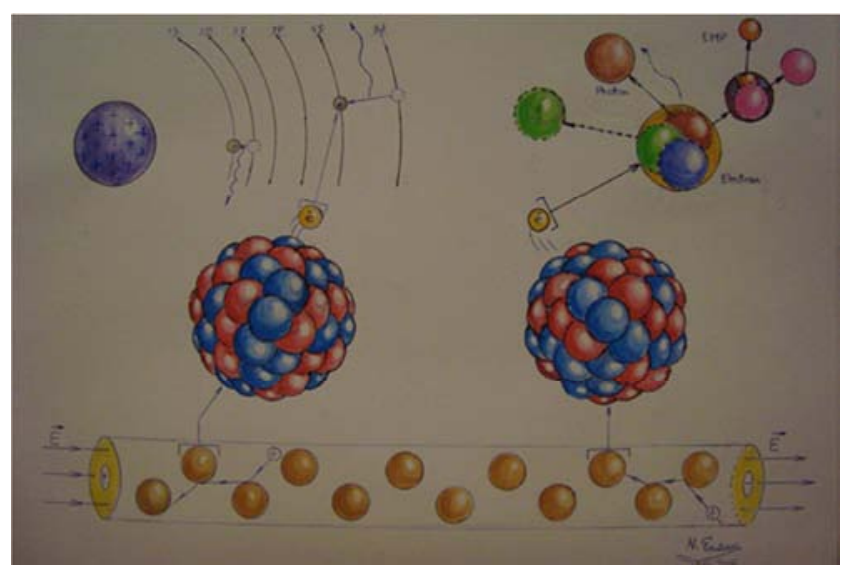

Figure 4. Drawn and painted by author Ensan. The tube in the below of the figure is a microscopic portion of an electric element made of Copper ${ }_{29}^{63} \mathrm{Cu}$. Already we know, there are exciting atoms because of striking energized free electrons by electric field of the circuit. Some electrons of these atoms which absorb energy move to upper orbits with higher distances from nucleus. At their return to the stable orbits, release photons (upper left of the figure). According to the result of this paper some electrons missing. It requires breaking to energy as the form of photons (upper right side). If electron is composed, it should be contained of at least two particles of like photons, etc. Although missing some electrons in the circuits is the main subject of this paper but photon also has a trace of electromagnetic wave. If photon after travelling a path and producing the electromagnetic field perpendicular to its direction of the travel and still maintain its properties as a photon, therefore it is composed. EMP (Electro-Magnetic Particle) could be one of its composition (up right of figure 4). 


\section{Summary and Conclusion}

We see in this paper that the tests show the current of one side (negative) at a circuit element like LED or resistor is different than the other side (positive) in a single loop. The Conservation of Charge Law as one of the principles of Classical Physics is incompatible with the observed mentioned experiments with measurements of higher resolution. For more accurate results, digital Multi meters with Ranges of ( $\mu \mathrm{A}, \mathrm{n} \mathrm{A}, .$.$) with Higher Resolutions and$ Better Accuracy is suggested. The tests can be adopted as an assignment for related courses at the Universities and Technical Colleges lab. The method, material list, and also precautions have been mentioned in the paper. Other cautions and safety measures must be added to the lab order, especially by increasing the circuit voltage and current.

Although based on classical Physics principles could not be a well that electrons fall down it, but is considered that a fraction of electrons missing. The possibility of leakage current is tested in LED light, but no measureable leaking amperage viewed. The interpretation could be that: A large number of missing electrons break to photons and it is happened not just in a sophisticated electron accelerator, but in daily life electric circuit. Electrons break although the drift velocity of electrons is very low. Sensing the hot wire (the wire which carries electricity) without cutting its insulator and only by circuit alert device or clamp ammeter could be the result of breaking some passing electrons and radiating photons (Photons produce Electromagnetic Waves) to the surrounding area.

\section{References}

[1] Arezu Jahanshir, Quanto-Optical Effects of Exciton-Polariton System, Buein Zahra Technical University, Iran, Published in The American Journal of Optics and Photonics, 2015 $\mathrm{http}: / /$ article.sciencepublishinggroup.com/html/10.11648.j.ajo p.20150305.16.html.
[2] Edwin Zong, The Unification of Mass and Energy Oasis Medical Group Inc., USA, Published in The American Journal of Physics and Applications, 2015

$\mathrm{http}: / /$ article.sciencepublishinggroup.com/html/10.11648.j.ajp a.20150305.13.html.

[3] Hugh D. Young, Roger A. Freedman, Sears and Zemansky's University Physics, Twelfth Edition, Pearson Education, Published by Dorling Kindersley, India, Pvt. Ltd. 2011, P1311.

[4] John D. Cutnell. Kenneth W. Johnson, Introduction to PHSICS, $8^{\text {th }}$ Edition, John Wiley \& Sons (Asia) Pte Ltd. Printed in Asia, 2010, P600.

[5] Giambattista Richardson Richardson, Physics, Published by McGraw-Hill Company, New York, United States of America, 2008, P639.

[6] PAUL A. TIPLER, GENE MUSCA, PHYSICS For Scientists and Engineers, Sixth Edition, W. H. Freeman and Company, Printed in The United States of America, Second Printing, 2008, P860.

[7] Paul A. Tipler, Ralph A. Llewellyn, MODERN PHYSICS, Fourth Edition, W. H. Freeman and Company, Printed in The United States of America, Second Printing, 2003, P450-451.

[8] FREDERICK J. BUECHE, Theory and Problems of COLLEGE PHYSICS, 7/ed, Schaum's Outline Series, McGRAW-HILL COMPANY, Printed in The United States of America, 1979, P210.

[9] Frederick J. Bueche, INTRODUCTION TO PHYSICS FOR SCIENTISTS AND ENGINEERS, McGRAW-HILL INTERNATIONAL EDITIONS, Printed in The United States of America, Singapore 1986, P859.

[10] Arthur Beiser, CONCEPT OF MODERN PHYSICS, McGRAW-HILL INTERNATIONAL EDITIONS, Printed in Singapore 1988, p524

[11] FRANCIS A. JENKINS, HARVEY E. WHITE, Fundamentals of Optics, Fourth Edition, McGRAW-HILL INTERNATIONAL EDITIONS, Printed in Singapore 1987, p524 P 698-704.

[12] Harris Hemmerling, INTRODUCTORY APPLIED PHYSICS, McGraw-Hill Company, Printed in Korea, 1981, P528-530. 